\title{
Impact of Physician Awareness on Diagnosis of Fetomaternal Hemorrhage
}

\author{
Annemarie Stroustrup ${ }^{\mathrm{a}-c}$ Callie Plafkin ${ }^{\mathrm{a}}$ David A. Savitz $^{\mathrm{d}, \mathrm{e}}$ \\ ${ }^{a}$ Icahn School of Medicine at Mount Sinai, ${ }^{b}$ Division of Newborn Medicine, Department of Pediatrics and \\ 'Department of Preventive Medicine, Mount Sinai Medical Center, New York, N.Y., and Departments of \\ dEpidemiology and ${ }^{\text {e } O b s t e t r i c s}$ and Gynecology, Brown University, Providence, R.I., USA
}

\author{
Key Words \\ Fetomaternal hemorrhage $\cdot$ Neonatal anemia $\cdot$ Perinatal \\ epidemiology
}

\begin{abstract}
Background: Fetomaternal hemorrhage (FMH) is a poorly understood condition in which the placenta allows transmission of fetal whole blood to the mother. FMH can cause fetal anemia resulting in critical illness, death or lifelong disability. Ascertainment of the incidence of FMH is limited by reliance on retrospective studies that are dependent on a diagnosis of FMH being made at the time of patient presentation. $\mathbf{O b}$ jective: To determine whether the diagnosis of $\mathrm{FMH}$ is made more frequently after an educational intervention to increase physician awareness of the condition. Methods: This is a retrospective cohort study of all neonates born at our institution from 1988 through 2010. The medical records of all neonates diagnosed with anemia in the first $24 \mathrm{~h}$ of life were reviewed. The incidence of FMH as a documented etiology of anemia was compared between infants born before and after our educational intervention. Results: Of 124,738 births during the study period, 572 neonates with neonatal anemia were identified. A total of 23 cases of FMH demonstrated by positive Kleihauer-Betke testing occurred in our cohort. The incidence of diagnosed FMH prior to our intervention was 22 per 1,000 anemic neonates compared to 182 per 1,000 afterwards $(p<0.001)$, while the incidence of neonatal anemia remained unchanged $(p=0.377)$. Conclusions:
\end{abstract}

FMH may be a significant cause of neonatal anemia. Diagnosis of $\mathrm{FMH}$ is highly dependent on physician awareness of the condition. Incorrect or absent diagnosis of the etiology of neonatal anemia has significant implications for our understanding of the epidemiology of FMH. $\quad$ ○ 2014 S. Karger AG, Basel

\section{Introduction}

A healthy placenta permits transfer of dissolved nutrients, gasses and waste products between the mother and fetus while keeping the cellular components of the two circulations separate. Fetomaternal hemorrhage (FMH) occurs when the normal flow of blood within the placenta is disrupted and fetal whole blood is transferred into the maternal circulation. It is common for the placental filter to 'leak' during normal pregnancy and delivery, resulting in the transfer of small volumes of fetal whole blood into the maternal bloodstream. Volumes of fetal blood thought to be clinically insignificant to the neonate can be detected after delivery in $50-75 \%$ of pregnancies that produce a healthy neonate $[1,2]$. Detection of fetal blood in the maternal circulation depends on the amount of blood transferred, the timeframe over which the hemorrhage occurs and the presence or absence of maternal antibodies to fetal erythrocytes [3]. Moderate-to-severe FMH is less common, occurring in an estimated 1-3 per 1,000 live births $[4,5]$ and causing an estimated $14 \%$ of fetal deaths [6]. FMH clearly occurs over a spectrum, and

\section{KARGER}

E-Mail karger@karger.com

www.karger.com/neo
(C) 2014 S. Karger AG, Basel

$1661-7800 / 14 / 1054-0250 \$ 39.50 / 0$
Annemarie Stroustrup, MD, MPH

Division of Newborn Medicine, Department of Pediatrics

Mount Sinai Medical Center, 1 Gustave L. Levy Place

Box 1508, New York, NY 10029 (USA)

E-Mail annemarie.stroustrup@mssm.edu 
the severity of FMH has historically been classified by the volume of fetal blood loss rather than the severity of illness of the neonate $[7,8]$. Although the exact volume of blood transfer needed to classify FMH as 'severe' is debated [9], increasing volumes of hemorrhage lead to increasing rates of adverse perinatal outcome.

Estimates of the incidence of severe FMH vary and probably do not reflect the reality of modern pregnancies, both because no prospective comprehensive epidemiological studies of the condition have been completed and because use of available testing for the condition is highly physician dependent. In fact, the only recent large-scale epidemiological evaluation of the condition concluded that discrepant rates of ordering laboratory evaluation for FMH in different populations, rather than underlying demographic predisposition for the condition, are likely to have accounted for a significant proportion of the demographic associations identified [5].

In the absence of known epidemiological risk factors for FMH or a comprehensive antenatal screening program, neonatal care providers must be vigilant to include FMH in their differential diagnosis when presented with an anemic neonate. Diagnosis of FMH requires specific blood testing of the mother - the Kleihauer-Betke (KB) acid elution test or flow cytometry for fetal cells in the maternal circulation - not routinely performed in the perinatal period. If perinatal care providers do not order FMH testing in response to neonatal anemia identified soon after birth, the diagnosis is missed. Making the correct diagnosis of FMH is important for risk stratification of the affected neonate, family planning and increased obstetric surveillance in future pregnancies, as well as in establishing epidemiological predictors for the condition.

In this study, we hypothesized that FMH is under-diagnosed in the neonatal anemic population. We further hypothesized that a simple educational intervention performed to raise awareness among neonatologists of FMH as a cause of neonatal anemia significantly impacted the diagnosis of FMH at our institution. To test these hypotheses, we conducted a retrospective study of all neonates diagnosed with anemia in the first $4 \mathrm{~h}$ after birth, comparing the incidence of FMH diagnosis before and after the educational intervention.

\section{Methods}

This is a retrospective cohort study of all neonates born at our institution from January 1, 1988 through December 31, 2010. Study subjects were identified from a database of clinical information prospectively maintained for all neonates admitted to the newborn medicine service at our institution (level I-IIIC nurseries). Anemic neonates were identified by a documented hematocrit $<50 \%$ within the first $4 \mathrm{~h}$ of life. Medical records from anemic neonates and their mothers were extracted for demographic and clinical information including any identified etiology of neonatal anemia. Each neonate's hematocrit was compared to published normal gestational age ranges $[10,11]$. Only neonates with hematocrit 1 standard deviation below the mean for gestational age were considered to have anemia and were included in subsequent analyses. Throughout this report, the terms 'neonatal anemia' and 'anemia' relate to low hemoglobin/hematocrit diagnosed in the first $4 \mathrm{~h}$ of life, and not to anemia diagnosed later that could be related to phlebotomy, poor hematopoiesis in preterm infants or other causes arising in the postnatal period. Of note, hematocrit testing is only performed at our institution for neonates with clinical indication, so anemic neonates who have no history or presentation triggering a complete blood count or hematocrit measurement are not identified.

The educational intervention consisted of two educational sessions of our institution's neonatology service in which the current literature on FMH incidence, diagnosis and prognosis were presented. Specifically, the definition of neonatal anemia by gestational age, necessary laboratory testing to make a diagnosis of $\mathrm{FMH}$, clinical implications of a diagnosis of $\mathrm{FMH}$, and neonatal outcomes associated with FMH were reviewed with all practicing neonatologists and neonatal-perinatal medicine fellows. These sessions occurred during a 2 -week period in January 2008. Potential study subjects born during this 2 -week window were excluded from analyses.

Subjects were grouped based on date of birth preceding or following this educational intervention. Patients born prior to the educational intervention were further grouped into 2- or 3-year epochs by date of birth for time trend comparison with patients born in the 3-year period after the intervention. Study subjects were considered to be affected by FMH if they had anemia for gestational age within the first $4 \mathrm{~h}$ following birth and their mother had positive $\mathrm{KB}$ testing during the birth hospitalization. KB testing was the only laboratory method for diagnosis of FMH available at our institution during the study period. The level of detection of $\mathrm{KB}$ testing at our institution is $0.1 \mathrm{ml}$ of fetal blood in the maternal circulation. If the $\mathrm{KB}$ assay is positive, the result is presented as a percentage of fetal blood detected in the maternal sample. No clinical interventions to increase FMH diagnosis besides our educational intervention (e.g. change in order templates, educational intervention with the obstetrical staff, etc.) were made during the study period. Group comparisons were made by Student's t test, analysis of variance (ANOVA) and $\chi^{2}$ test as appropriate. This study was approved by the Icahn School of Medicine Program for the Protection of Human Subjects.

\section{Results}

Of 124,738 newborn admissions during the study period, approximately $41 \%$ had a hematocrit measured in the first $24 \mathrm{~h}$ of life. Of these, 572 neonates $(0.5 \%$ of all births) had anemia diagnosed in the first $4 \mathrm{~h}$ of life. In the 20 years prior to our intervention, 506 cases of ane- 
mia or 4.8 per 1,000 births were identified. In the first 3 years following our intervention, there were 66 cases of anemia or 3.6 per $1,000(\mathrm{p}=0.377)$. Neonates with anemia born before and after our educational intervention were compared for basic demographic and clinical characteristics. Discrepancy in ethnicity was found between the earlier and later cohorts, with more white patients in the earlier cohort and fewer patients with unknown ethnicity in the later cohort $(\mathrm{p}=0.003)$. There was no significant difference in birth weight or gestational age between cohorts.

For the majority of neonates with identified neonatal anemia, the cause of anemia was unknown at the time of discharge from birth hospitalization. Comparisons were made across the entire study population between anemic patients for whom $\mathrm{KB}$ testing was conducted and those for whom such testing was not conducted. No significant ethnic difference was found between patients whose mothers received $\mathrm{KB}$ testing in response to neonatal anemia and those who did not. Birth weight and gestational age were significantly greater among neonates for whom KB testing was completed (2,616 \pm 804 vs. $2,006 \pm 1,015 \mathrm{~g}$; $35.8 \pm 3.8$ vs. $32.4 \pm 5.4$ weeks; $\mathrm{p}<0.001$ for each comparison).

A total of 23 cases of FMH occurred in the study population. The incidence of diagnosed FMH prior to our intervention was 22 per 1,000 anemic neonates compared to 182 per 1,000 afterwards ( $<<0.001$; fig. 1 ). Basic demographic and perinatal clinical information for pregnancies diagnosed with $\mathrm{FMH}$ by $\mathrm{KB}$ testing before and after our intervention is presented in table 1 . Birth weight for anemic neonates born following FMH ranged from 1,305 to $4,320 \mathrm{~g}$, and gestational age at birth ranged from $29(1 / 7)$ to $40(3 / 7)$ weeks. All were singleton pregnancies. No mother of a neonate with FMH had a history of illicit drug use or antenatal trauma. KB testing demonstrated $0.05-8 \%$ of fetal cells in the maternal circulation. Overall, 3 neonates (13\%) born following FMH died before discharge from the neonatal intensive care unit, and 1 (4\%) was discharged to a long-term care facility. The rest were discharged home. Duration of neonatal intensive care unit hospitalization ranged from 1 to 61 days. Only birth weight differed significantly before and after the educational intervention.

Using the incidence of FMH in our later cohort as a minimum incidence of the condition in our study population, we extrapolated the number of missed cases in the earlier cohort (fig. 2). We estimate that the diagnosis was probably missed in a minimum of 81 cases over the 20 -year period prior to our educational intervention.

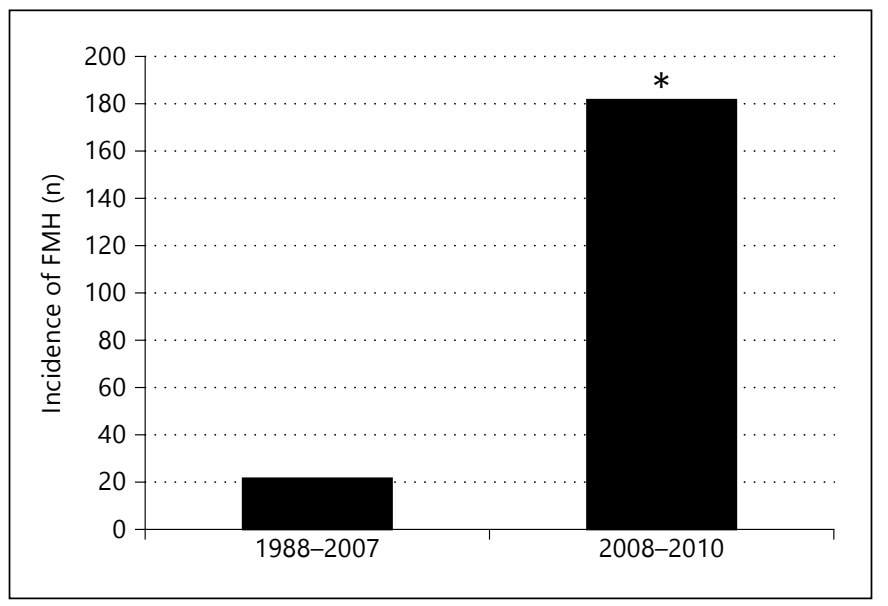

Fig. 1. Incidence of FMH per 1,000 anemic neonates. Incidence of $\mathrm{KB}$-proven $\mathrm{FMH}$ diagnosis associated with neonatal anemia before and after an educational intervention to raise neonatologist awareness of the diagnosis. There was a significant rise in incidence of diagnosis in the later time period $\left({ }^{*} \mathrm{p}<0.001\right)$.

\section{Discussion}

In utero signs of fetal anemia including decreased fetal movement and sinusoidal fetal heart rate tracing or devastating neonatal illness due to severe anemia at birth may prompt the astute clinician to test the postpartum mother for fetal cells in the maternal circulation. In this study, we demonstrate that a simple educational intervention can substantially increase the incidence of FMH diagnosis among anemic neonates. Although a comprehensive prospective study is needed to determine the true incidence of FMH among patients with neonatal anemia, this study implies that FMH is a commonly missed diagnosis.

The only recent large-scale epidemiological surveys of FMH focus on neonates with severe anemia $[5,12]$. However, even mild anemia in infancy has documented clinical significance including poor growth and cognitive deficit [13]. Despite these consequences and a suggested recent rise in incidence of severe FMH [9], early predictors of FMH remain unknown and no cause of hemorrhage can be identified in over $80 \%$ of cases [9]. Maternal, pregnancy or neonatal risk factors appropriate for early screening or diagnosis of FMH in the general population have not been elucidated $[14,15]$.

Our study indicates that reflex KB testing in the face of even low-level neonatal anemia can identify cases of FMH that would otherwise be missed. It also indicates that FMH may be a far more common cause of neonatal anemia than previously reported. This study also indicates 
Table 1. Perinatal characteristics when fetomaternal hemorrhage diagnosed

\begin{tabular}{|c|c|c|c|}
\hline & $\begin{array}{l}1988-2007 \\
(n=11)\end{array}$ & $\begin{array}{l}2008-2010 \\
(\mathrm{n}=12)\end{array}$ & $\mathrm{p}$ \\
\hline \multicolumn{4}{|c|}{ Demographic and anthropomorphic characteristics } \\
\hline Male & $2(18)$ & $5(42)$ & 0.37 \\
\hline \multicolumn{4}{|l|}{ Ethnicity } \\
\hline White & $8(73)$ & $8(67)$ & 0.92 \\
\hline Black & $1(9)$ & $1(8)$ & \\
\hline Hispanic & $1(9)$ & $1(8)$ & \\
\hline Asian & $1(9)$ & $1(8)$ & \\
\hline Other & $0(0)$ & $1(8)$ & \\
\hline Unknown & $8(73)$ & $8(67)$ & \\
\hline Birth weight, $g$ & $2,450 \pm 659$ & $3,088 \pm 583$ & 0.02 \\
\hline Gestational age, weeks & $35.5 \pm 4.0$ & $37.5 \pm 2.8$ & 0.17 \\
\hline SGA & $0(0)$ & $2(17)$ & 0.48 \\
\hline Private insurance & $11(100)$ & $10(83)$ & 0.48 \\
\hline \multicolumn{4}{|c|}{ Maternal and pregnancy characteristics } \\
\hline Maternal diabetes & $1(9)$ & $0(0)$ & 0.48 \\
\hline Maternal tobacco use & $1(9)$ & $0(0)$ & 0.48 \\
\hline Maternal hypercoagulability & $1(9)$ & $0(0)$ & 0.48 \\
\hline Maternal fibroids & $1(9)$ & $1(8)$ & 1.00 \\
\hline Placenta previa & $1(9)$ & $0(0)$ & 0.48 \\
\hline Oligohydramnios & $0(0)$ & $1(8)$ & 1.00 \\
\hline \multicolumn{4}{|l|}{ Peripartum characteristics } \\
\hline Maternal hemoglobin, g/dl & $11.2 \pm 1.3$ & $12.0 \pm 1.7$ & 0.21 \\
\hline Maternal hematocrit, \% & $33.9 \pm 2.1$ & $35.1 \pm 5.1$ & 0.49 \\
\hline Emergent cesarian delivery & $4(36)$ & $9(75)$ & 0.10 \\
\hline Meconium at delivery & $1(10)$ & $6(50)$ & 0.07 \\
\hline Placental abruption & $3(27)$ & $0(0)$ & 0.21 \\
\hline Abnormal fetal heart tracing & $4(36)$ & $8(67)$ & 0.22 \\
\hline Resuscitation at delivery & $5(46)$ & $5(42)$ & 1.00 \\
\hline Apgar at $1 \mathrm{~min}$ & $7(4-9)$ & $8(5-9)$ & 0.41 \\
\hline Apgar at $5 \mathrm{~min}$ & $9(7-9)$ & $8(7-9)$ & 0.68 \\
\hline Kleihauer-Betke, \% & $2.4 \pm 1.6$ & $3.0 \pm 2.7$ & 0.62 \\
\hline \multicolumn{4}{|l|}{ Neonatal outcomes } \\
\hline First neonatal hemoglobin, g/dl & $7.18 \pm 3.4$ & $7.17 \pm 2.4$ & 0.99 \\
\hline First neonatal hematocrit, $\%$ & $21.6 \pm 9.1$ & $21.8 \pm 7.0$ & 0.93 \\
\hline First neonatal reticulocyte, $\%$ & $8.8 \pm 5.4$ & $9.8 \pm 6.3$ & 0.71 \\
\hline Peak bilirubin, mg/dl & $10.6 \pm 6.0$ & $10.9 \pm 4.9$ & 0.88 \\
\hline Blood transfusions & $1(0-2)$ & $2(1-3)$ & 1.00 \\
\hline Central line placed & $6(55)$ & $7(58)$ & 1.00 \\
\hline $\mathrm{DIC}$ & $1(9)$ & $0(0)$ & 0.48 \\
\hline Asphyxia or HIE & $3(27)$ & $2(17)$ & 0.64 \\
\hline Seizures & $2(18)$ & $1(8)$ & 0.59 \\
\hline Hypotension & $3(27)$ & $5(42)$ & 0.67 \\
\hline PFC & $2(18)$ & $1(8)$ & 0.59 \\
\hline $\mathrm{PPV}, \mathrm{h}$ & $31(0-0)$ & $0(0-18)$ & 0.37 \\
\hline Died before NICU discharge & $1(9)$ & $2(17)$ & 1.00 \\
\hline
\end{tabular}




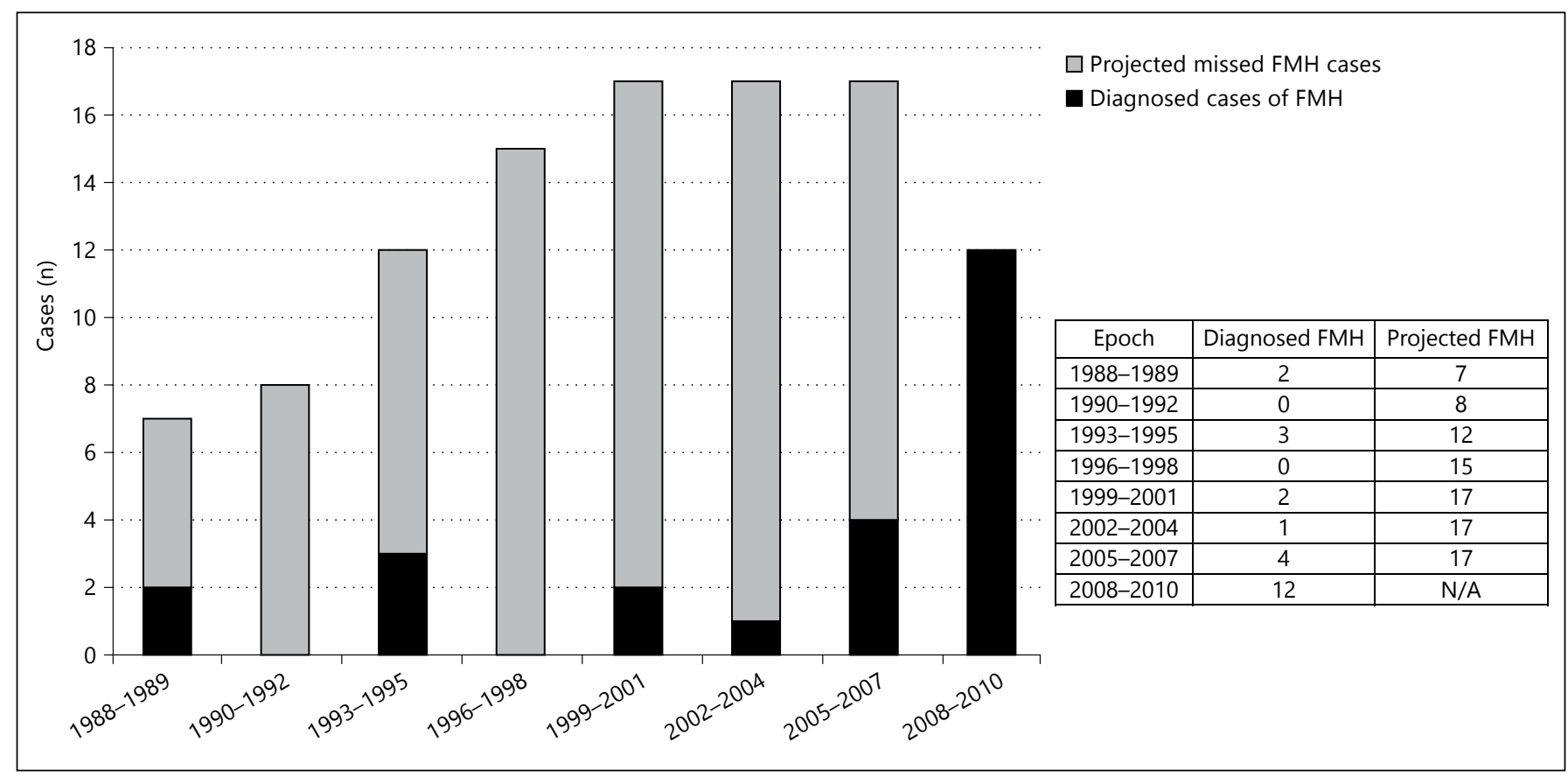

Fig. 2. Diagnosed and projected cases of FMH. Diagnosis of FMH after an intervention to increase physician awareness of the condition was significantly more common than prior to intervention. The incidence of FMH in the last epoch was used to estimate the minimum number of missed cases in earlier epochs. The number of FMH cases predicted by the 2008-2010 incidences far exceeds the number of cases actually detected in earlier epochs.

tional age norms, this may indicate an opportunity to further educate neonatologists about the definitions of anemia in the preterm population.

Our study also highlights the high morbidity and mortality associated with FMH. We found a 13\% mortality and significant morbidity in our population with FMH. Of note, only $52 \%$ of pregnancies were marked by an abnormal intrapartum fetal heart rate tracing and only $57 \%$ were delivered by emergent cesarean delivery. This may indicate a specific opportunity to educate neonatal care providers that FMH may commonly occur in the absence of typical markers of intrapartum distress.

Most significantly, our findings highlight that conclusions drawn from cohort studies based on the diagnoses of FMH made in an ad hoc fashion may offer a highly skewed perspective of the incidence and epidemiology of $\mathrm{FMH}$, as it is likely that they miss a significant number of patients affected by FMH. Thus, epidemiological conclusions drawn from clinical cohorts may be inaccurate.

Our study has a number of limitations. It is a singlecenter study of a rare condition - or at least a rarely diagnosed condition - so generalizability may be limited. Although there is a correlation between an educational intervention for our neonatology group and the incidence 
of identified FMH in our NICU, this correlation does not prove causation. It is possible that other factors beyond our educational intervention prompted an increased awareness of FMH among our physicians. To address this possibility, we evaluated the published literature indexed in PubMed from 1988 through 2010, and calculated an annual incidence of English language publications (print or Epub ahead of print) identified by the search phrase 'fetomaternal hemorrhage'. From 1988 to 2007 there were 480 such publications and from 2008 to 2010 there were 72 - an identical incidence for each time period of 24 FMH publications per year. Although the incidence of topical publications during the 2 time epochs was identi$\mathrm{cal}$, it is possible that the new literature in the later time period impacted the diagnosis rate of FMH in our unit, independent of our educational intervention.

We also note that the educational intervention did not include specific screening guidelines, so case identification in the last epoch was still dependent on physician awareness of the condition and willingness to order $\mathrm{KB}$ testing. Additionally, our intervention did nothing to impact the diagnosis of neonatal anemia itself, so additional cases of FMH causing asymptomatic anemia were certainly missed. Our incidence of FMH in the postintervention time period, despite being 60 -fold higher than found in studies relying on existing clinical cohorts, possibly underestimates the incidence of FMH causing fetal anemia.
Clearly, a well-designed prospective study is needed to both accurately estimate the incidence of FMH and describe the affected population.

\section{Conclusions}

FMH may be a significant cause of neonatal anemia. Diagnosis of FMH is highly dependent on physician awareness. If physicians do not order $\mathrm{KB}$ or other definitive testing for $\mathrm{FMH}$ in response to neonatal anemia, the condition will go undetected. This has significant impact on a physician's ability to provide an accurate prognosis for anemic neonates and their families, as well as for our general understanding of the epidemiology of FMH.

\section{Acknowledgment}

This study was supported by grant UL1TR000069 from the National Center for Advancing Translational Sciences, National Institutes of Health (KL2RR029885 to Dr. Stroustrup). The funding agency had no role in study design or execution.

\section{Disclosure Statement}

The authors have no conflicts of interest to disclose.

\section{References}

$>1$ Bowman JM, Pollock JM, Penston LE: Fetomaternal transplacental hemorrhage during pregnancy and after delivery. Vox Sang 1986; 51:117-121.

-2 Sebring ES, Polesky HF: Fetomaternal hemorrhage: incidence, risk factors, time of occurrence, and clinical effects. Transfusion 1990; 30:344-357.

$>3$ Larsen R, Berkowicz A, Lousen T, Hedegaard M, Clausen FB, Krog GR, Dziegiel MH: Massive fetomaternal hemorrhage: clearance of fetal red blood cells after intravenous anti-D prophylaxis monitored by flow cytometry. Transfusion 2008;48:1707-1712.

$\checkmark 4$ de Almeida V, Bowman JM: Massive fetomaternal hemorrhage: Manitoba experience. Obstet Gynecol 1994;83:323-328.

5 Stroustrup A, Trasande L: Demographics, clinical characteristics and outcomes of neonates diagnosed with fetomaternal haemorrhage. Arch Dis Child Fetal Neonatal Ed 2012; 97:F405-F410.
-6 Laube DW, Schauberger CW: Fetomaternal bleeding as a cause for 'unexplained' fetal death. Obstet Gynecol 1982;60:649-651.

$\checkmark 7$ Wylie BJ, D’Alton ME: Fetomaternal hemorrhage. Obstet Gynecol 2010;115:1039-1051.

$>8$ Kecskes Z: Large fetomaternal hemorrhage: clinical presentation and outcome. J Matern Fetal Neonatal Med 2003;13:128-132.

$>9$ Giacoia GP: Severe fetomaternal hemorrhage: a review. Obstet Gynecol Surv 1997;52:372380.

-10 Alur P, Devapatla SS, Super DM, Danish E, Stern T, Inagandla R, Moore JJ: Impact of race and gestational age on red blood cell indices in very low birth weight infants. Pediatrics 2000;106:306-310.

$>11$ Jopling J, Henry E, Wiedmeier SE, Christensen RD: Reference ranges for hematocrit and blood hemoglobin concentration during the neonatal period: data from a multihospital health care system. Pediatrics 2009;123:e333e337.
12 Christensen RD, Lambert DK, Baer VL, Richards DS, Bennett ST, Ilstrup SJ, Henry E: Severe neonatal anemia from fetomaternal hemorrhage: report from a multihospital health-care system. J Perinatol 2013;33:429434.

13 De-Regil LM, Jefferds ME, Sylvetsky AC, Dowswell $\mathrm{T}$ : Intermittent iron supplementation for improving nutrition and development in children under 12 years of age. Cochrane Database Syst Rev 2011;12: CD009085.

14 David M, Smidt J, Chen FC, Stein U, Dudenhausen JW: Risk factors for fetal-tomaternal transfusion in $\mathrm{Rh} \mathrm{D}$-negative women - results of a prospective study on 942 pregnant women. J Perinat Med 2004; 32:254-257.

15 Samadi R, Greenspoon JS, Gviazda I, Settlage RH, Goodwin TM: Massive fetomaternal hemorrhage and fetal death: are they predictable? J Perinatol 1999;19:227-229. 